\title{
Synonymous but not the same: the causes and consequences of codon bias
}

\section{Joshua B. Plotkin* and Grzegorz Kudla}

\begin{abstract}
Despite their name, synonymous mutations have significant consequences for cellular processes in all taxa. As a result, an understanding of codon bias is central to fields as diverse as molecular evolution and biotechnology. Although recent advances in sequencing and synthetic biology have helped to resolve longstanding questions about codon bias, they have also uncovered striking patterns that suggest new hypotheses about protein synthesis. Ongoing work to quantify the dynamics of initiation and elongation is as important for understanding natural synonymous variation as it is for designing transgenes in applied contexts.
\end{abstract}

When the inherent redundancy of the genetic code was discovered, scientists were rightly puzzled by the role of synonymous mutations ${ }^{1}$. The central dogma of molecular biology suggests that synonymous mutations - those that do not alter the encoded amino acid - will have no effect on the resulting protein sequence and, therefore, no effect on cellular function, organismal fitness or evolution. Nonetheless, in most sequenced genomes, synonymous codons are not used in equal frequencies. This phenomenon, termed codon-usage bias (FIG. 1), is now recognized as crucial in shaping gene expression and cellular function through its effects on diverse processes, ranging from RNA processing to protein translation and protein folding. Naturally occurring codon biases are pervasive, and they can be extremely strong. Some species such as Thermus thermophilus avoid certain codons almost entirely. Synonymous mutations are important in applied settings as well - the use of particular codons can increase the expression of a transgene by more than 1,000 -fold ${ }^{2}$.

We already enjoy a broad array of often conflicting hypotheses for the mechanisms that induce codonusage biases in nature, and for their effects on protein synthesis and cellular fitness. Until recently, we have been unable to systematically interrogate these hypotheses through large-scale experimentation. As a result, despite decades of interest and substantial progress in understanding codon-usage biases, there is an overabundance of plausible explanatory models whose relative, quantitative contributions are seldom compared.
Advances in synthetic biology, mass spectrometry and sequencing now provide tools for systematically elucidating the molecular and cellular consequences of synonymous nucleotide variation. Such studies have refined our understanding of the relative roles of initiation, elongation, degradation and misfolding in determining protein expression levels of individual genes and the overall fitness of a cell. This information, in turn, is helping researchers to distinguish among the forces that shape naturally occurring patterns of codon usage. Researchers can also leverage high-throughput studies in applied settings that require controlled, heterologous gene expression, for example, to improve design principles for vaccine development and gene therapy.

Here we review the causes, consequences, and practical use of codon-usage biases. Because we already benefit from several outstanding reviews on naturally occurring codon biases ${ }^{3-7}$, we focus here on those classical hypotheses that remain unresolved and the recent developments arising from high-throughput studies. We begin by summarizing the empirical patterns of codon usage that are observed across species, across genomes and across individual genes. We describe the diverse array of mechanistic hypotheses for the causes of such variation and the sequence signatures that support them. Against this backdrop of hypotheses and sequence analysis, we describe experimental work that relates codon usage to endogenous gene expression and cellular fitness. From this, we turn to experimental studies on heterologous gene expression and their implications both for understanding 


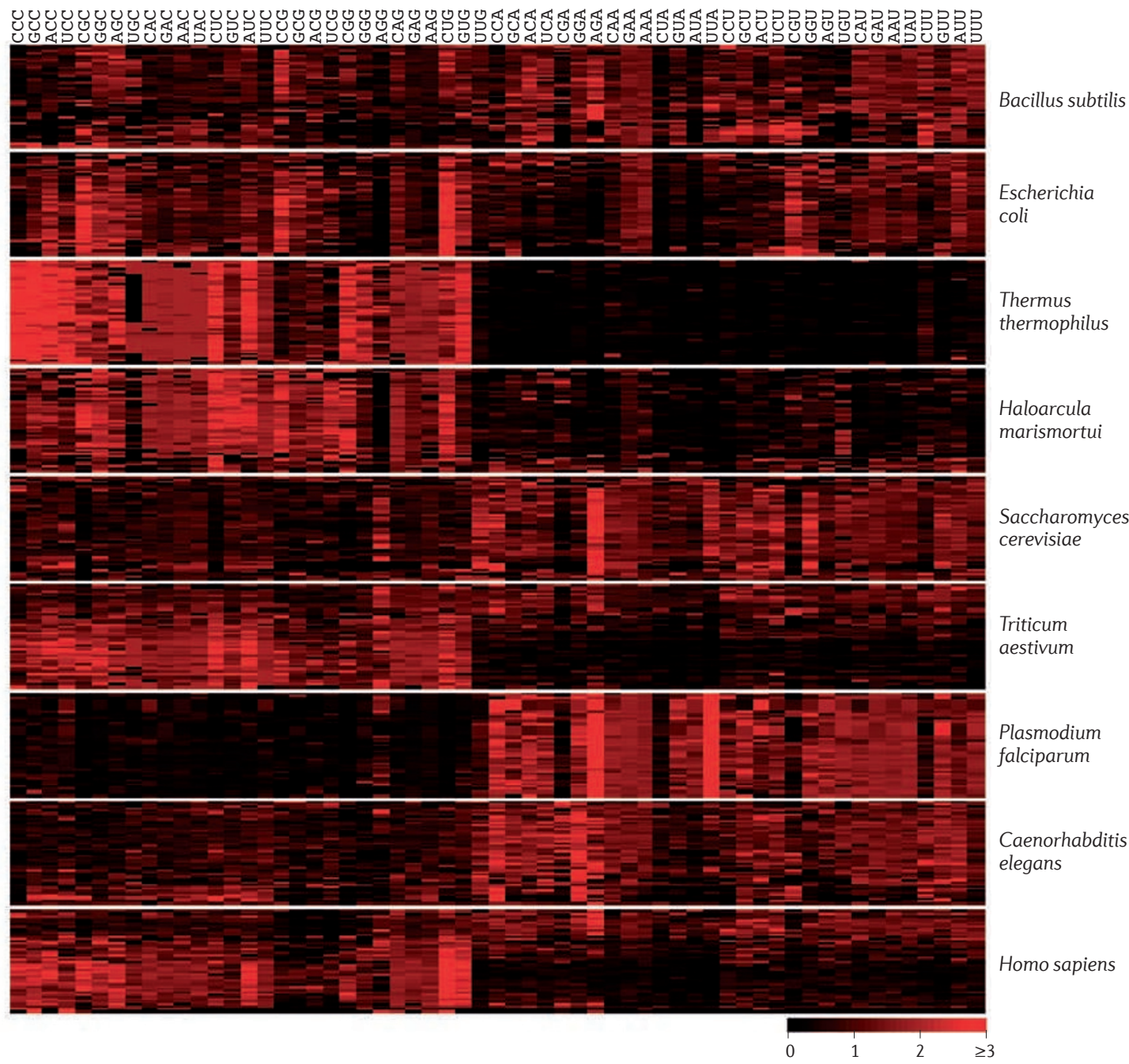

Figure 1 | Codon bias within and between genomes. The relative synonymous codon usage (RSCU) ${ }^{127}$ is plotted for 50 randomly selected genes from each of nine species. RSCU ranges from 0 (when the codon is absent), through 1 (when there is no bias) to 6 (when a single codon is used in a six-codon family). Methionine, tryptophan and stop codons are omitted. Genes are in rows and codons are in columns, with C-and G-ending codons on the left side of each panel. Note the extensive heterogeneity of codon usage among human genes. Other measures of a gene's codon bias include the codon adaptation index (CAl; the similarity of codon usage to a reference set of highly expressed genes $)^{35}$, the frequency of 'optimal' codons (FOP) ${ }^{28}$ and the tRNA adaptation index (tAl; the similarity of codon usage to the relative copy numbers of tRNA genes) $)^{128}$.

natural synonymous variation and for engineering new constructs in applied settings.

\section{Mechanistic hypotheses}

Significant deviations from uniform codon choice have been observed in species from all taxa, including bacteria, archaea, yeast, fruitflies, worms and mammals. The overall codon usage in a genome can differ dramatically between species, although seldom between closely related species ${ }^{6}$.

Mutational versus selective hypotheses. Explanations for patterns of codon usage, within or between species, fall into two distinct categories that are associated with two independent forces in molecular evolution: mutation and natural selection ${ }^{3-5}$.
A mutational explanation posits that codon bias arises from the properties of underlying mutational processes - for example, biases in nucleotides that are produced by point mutations ${ }^{8}$, contextual biases in the point mutation rates or biases in repair. Mutational explanations are neutral because they do not require any fitness advantage or detriment to be associated with alternative synonymous codons. Mutational mechanisms are typically invoked to explain interspecific variation in codon usage, especially among unicellular organisms.

Explanations involving natural selection posit that synonymous mutations somehow influence the fitness of an organism, and they can therefore be promoted or repressed throughout evolution. Selective mechanisms are typically invoked to explain variation in codon usage across a genome or across a gene, although 


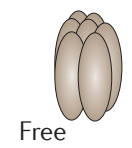

ribosomes
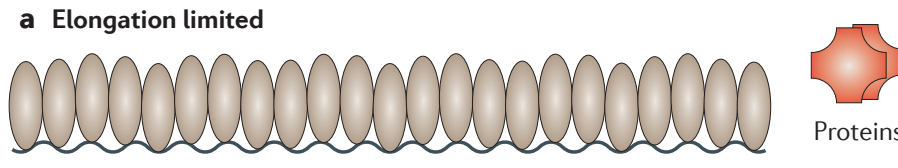

Proteins

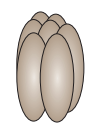

b Initiation limited, low codon adaptation
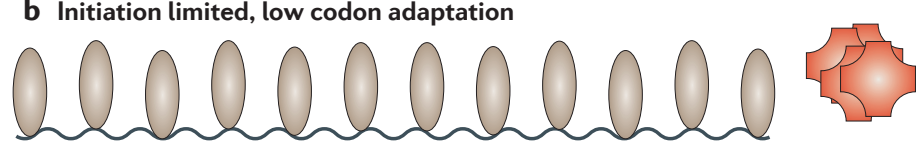

c Initiation limited, high codon adaptation
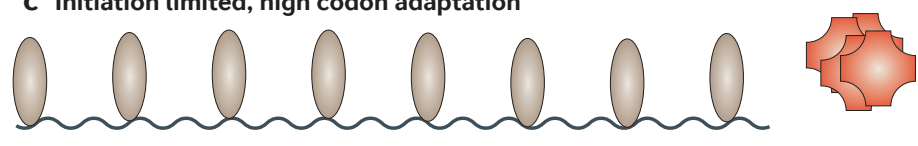

d Initiation limited (very slow), high codon adaptation
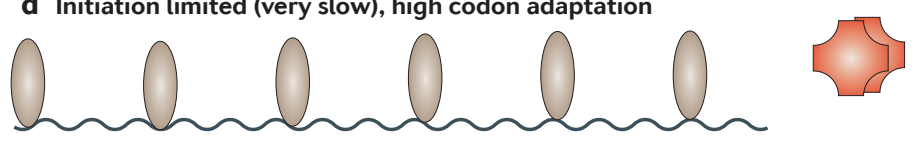

Figure 2 | Relationships between initiation rate, elongation rate, ribosome density and the rate of protein synthesis for endogenous genes. The steady-state rate of protein synthesis and density of ribosomes bound on an mRNA both depend on the rates of initiation and elongation. When elongation is the rate-limiting step in a gene's translation (case A), the message will be covered as densely as possible by ribosomes and faster elongation will tend to increase the rate of protein synthesis. However, most endogenous genes are believed to be initiation limited (cases B, C and D), so that their transcripts are not completely covered by ribosomes. This is evidenced by extensive variability in ribosome densities across endogenous mRNAs ${ }^{67}$. For two initiation-limited genes with the same initiation rate, the mRNA with faster elongation (afforded by, say, higher codon adaptation to tRNA pools) will have a lower density of translating ribosomes (C versus B) but no greater rate of termination. Thus, when initiation is limiting, high codon adaptation should not be expected to increase the amount of protein that is produced per mRNA molecule (protein amounts are the same in B and C). A lower density of ribosomes can also occur when two initiation-limited genes have the same elongation rate, but one has a slower initiation rate (D versus $C$ ). In this case, the amount of protein that is produced will be lower for the mRNA that has the slower initiation rate (D). The extent to which variation in ribosome densities ${ }^{67}$ arises from variation in initiation versus elongation rates remains to be determined. In all cases shown here, as is true for most endogenous genes, the gene's mRNA does not account for a substantial proportion of total cellular mRNA, so that the rates of initiation and elongation do not substantially alter the pool of free ribosomes (compare with FIG. 4).

some interspecific variation is also attributable to such mechanisms (see below).

Selective and neutral explanations for codon usage are not mutually exclusive, and both types of mechanisms surely have a role in patterning synonymous variation within and between genomes ${ }^{3,5,9}$. Below we discuss the patterns of codon usage that have been documented at various levels of biological organization in light of their mutational or selective causes.

\section{Patterns of codon usage}

Patterns across species. The strongest single determinant of codon-usage variation across species is genomic GC content. In fact, differences in codon usage between bacterial species can be accurately predicted from the nucle-
A subset of tRNAs that carry

the same amino acid.

\begin{abstract}
otide content in their non-coding regions ${ }^{3,10}$. Genomic
\end{abstract}
GC content is itself typically determined by mutational processes that act across the whole genome. As a result, most interspecific variation in codon usage is attributed to mutational mechanisms ${ }^{3,10}$, although the molecular causes of mutation biases are largely unknown ${ }^{10}$. Contrary to early expectations, the GC content of bacterial genomes or protein-coding genes is not correlated with optimal growth temperature (although, interestingly, structural RNAs show such a correlation $)^{11}$.

In those species for which the point mutation rate depends strongly on the sequence context of a nucleotide - for example, in mammals, which experience hypermutable CpG dinucleotides - the mutational model predicts a strong context dependence of codon usage, which has indeed been observed ${ }^{12}$. Thus, at the genomic scale, neutral processes that do not discriminate among synonymous mutations remain plausible for explaining interspecific variation in codon usage among higher eukaryotes and they are well accepted as the primary determinants of interspecific variation in most other taxa (but see REF. 13).

Aside from mutation biases, adaptation of codon usage to cellular tRNA abundances can also influence synonymous sequence variation across species (see below), as codon usage and tRNA regulation can coevolve. Finally, some neutral processes that are responsible for codon bias across taxa are not mutational per se. Even in the absence of selection at synonymous sites, selection at non-synonymous sites can induce differences in nucleotide composition between coding and non-coding regions $s^{5,14-16}$.

Patterns across a genome. There is often systematic variation in codon usage among the genes in a genome, usually attributed to selection. In organisms, including Escherichia coli, Saccharomyces cerevisiae, Caenorhabditis elegans, Arabidopsis thaliana, Drosophila melanogaster and possibly also mammals (see below), there is a significant positive correlation between a gene's expression level and the degree of its codon bias, and a negative correlation between expression level and the rate of synonymous substitutions between divergent species $^{9,17,18}$ features that are difficult to explain through mutation alone. Although mutational effects could possibly covary with expression levels, because transcription can be mutagenic ${ }^{19,20}$, this effect is unlikely to account for the correlations between codon usage and expression levels that are observed in numerous species ${ }^{5,19,21}$.

The classic explanation for systematic variation across a genome is selectionist: codon bias is more extreme in highly expressed genes to match a skew in iso-accepting tRNAs and, thereby, provide a fitness advantage through increased translation efficiency or accuracy of protein synthesis ${ }^{9,17,22-27}$. There is strong evidence for this hypothesis in several species, mostly in the form of broad correspondences between the 'preferred codons' that are used in highly expressed genes and measures of relative tRNA abundances ${ }^{28-32}$. As a result, translational selection remains the dominant explanation for systematic variation in codon usage among genes, despite the fact that supporting evidence is sometimes incomplete: direct 


\section{Box 1 Selection for accuracy or efficiency?}

The nature of translational selection remains a topic of active debate. Codons that are adapted to tRNA pools might be preferentially used in highly expressed genes, because such genes experience greater pressure for translational efficiency ${ }^{28,29,110}$, accuracy $22-27$ or both. Efficient elongation of a transcript might increase its protein yield ${ }^{2,36,37,92}$, or it may provide a global benefit to the cell by increasing the number of ribosomes that are available to translate other messages, even if it does not increase the yield of the transcript itself ${ }^{3,7,9,55}$. Accurate elongation, by contrast, benefits the cell by reducing the costs of useless mistranslation products or the toxicity of harmful mistranslation products ${ }^{111}$. These two models can make different predictions for the fitness costs of maladaptive codons, as a function of transcript level.

There are several lines of sequence-based evidence that discriminate between the efficiency and accuracy hypotheses. Some of the most compelling evidence in favour of accuracy was introduced by Akashi ${ }^{22,23,113}$, who found a greater tendency towards tRNA-adapted codons at residues that are strongly conserved across divergent Drosophila species; this suggests that sites that are under strong negative selection at the amino acid level also show stronger codon adaptation, presumably to reduce mistranslation. The same finding was later extended to Caenorhabditis elegans ${ }^{113}$ and unicellular organisms ${ }^{25,27}$. A separate line of evidence arises from the correlation between codon adaptation and gene length in Escherichia coli ${ }^{24}$, reflecting a greater energetic cost of missense and nonsense translation errors in a long protein, especially if they occur near the $3^{\prime}$ end. However, the relationship with gene length does not hold in C. elegans, Drosophila melanogaster or Arabidopsis thaliana ${ }^{21}$. Other evidence for the accuracy hypothesis comes from simulations of sequence evolution, protein translation and protein folding ${ }^{26}$.

There is also convincing evidence in favour of translational efficiency, especially in prokaryotes. The most compelling observation is a broad correlation between the minimum generation time of a bacterial species and the strength of selection it experiences for codon adaptation in highly expressed genes ${ }^{34,114}$. We would expect to see this correlation if preferred codons increase the elongation rate, which is beneficial for rapid growth, but it is unclear why we would observe this correlation if preferred codons increase only the accuracy of elongation. Furthermore, Zhang and others have recently shown that codon usage in highly expressed yeast genes is consistent with selection to avoid unnecessary ribosomal sequestration of messages (W. Qian, J. Yang, N. Pearson, C. Maclean and J. Zhang, personal communication).

The accuracy and efficiency hypotheses are not mutually exclusive, in general. However, in a recent computational study, Shah and Gilchrist ${ }^{115}$ demonstrated that codons corresponding to more abundant tRNAs are not always expected to produce lower missense error rates, as has been commonly assumed. Moreover, they found that, for some amino acids, pressure for elongation speed would result in a different codon choice than would pressure for elongation accuracy. Whether patterns of codon bias in evolutionarily conserved residues ${ }^{22}$ occurs only for those amino acids for which efficiency and accuracy selection have the same predicted effect on codon choice remains unresolved and might help to distinguish between these two modes of selection.

Negative selection

A form of natural selection

that suppresses alternative genetic variants in favour of the wild type.

Horizontal gene transfer The transfer of genetic material

from one species into another.

Isochore

A large fragment of a

chromosome that is

characterized by

homogeneous GC content. measurements of tRNA abundances are rare in higher eukaryotes; the correspondence of tRNA abundance with tRNA copy number ${ }^{5}$ is weak in D. melanogaster and humans $s^{33}$; and $30 \%$ of bacterial species show no evidence of translational selection ${ }^{34}$.

There are two possible directions of causality relating an endogenous gene's expression level and the degree of its codon adaptation ${ }^{35}$ to tRNA abundances. In one view $^{2,36,37}$, high codon adaptation induces strong protein expression, because rapid and/or accurate elongation increases a given protein's rate of synthesis; in the other view, strong expression selects for high codon adaptation to avoid costs that scale with a gene's expression level. In the biotechnology literature, the former interpretation is de rigueur, whereas in the literature on molecular evolution, the latter interpretation prevails ${ }^{3-5}$.
The idea that high codon adaptation induces high protein levels per mRNA molecule does not square well with the notion that initiation is generally rate limiting for endogenous protein production ${ }^{7,9,38,39}$ (although it may apply to heterologous genes (see below)). When initiation is limiting, the elongation rate should not influence the amount of protein that is produced from a given message $\mathrm{e}^{7,9}$ (FIG. 2). Moreover, from an evolutionary perspective, if high protein levels are desirable, it would seem easier to tune a promoter for increased transcription than to select on hundreds of individual synonymous mutations, each of which has only a marginal effect on the overall amount of protein synthesis. Conversely, the use of poorly adapted codons to slow the translation of genes expressed at low levels ${ }^{36}$ would seem wasteful compared to simply reducing transcription or slowing initiation.

Although evolutionary studies generally agree that high expression selects for high codon adaptation in endogenous genes (as opposed to the converse), the precise nature of fitness gains associated with translationally adapted codons remains a topic of active debate (BOX 1 ). Furthermore, even though translational efficiency is energetically beneficial to the cell, efficient translation generally increases the amount of cell-to-cell variation in expression levels ${ }^{40}$ and this noise is typically deleterious ${ }^{41}$. Although translational selection has received the most attention, systematic variation in codon usage across a genome can also be caused by neutral processes in certain species; these processes include horizontal gene transfer ${ }^{42}$, different nucleotide bias in leading and lagging strands of replication in bacteria ${ }^{43}$ and isochore structure in mammals (BOX 2).

Patterns across a gene. Codon usage can vary dramatically even within a single gene. Synonymous mutations at specific sites may experience selection because they disrupt motifs that are recognized by transcriptional or by post-transcriptional regulatory mechanisms, for example, microRNAs. Sites that require ribosomal pausing for proper co-translational protein folding or ubiquitin modification ${ }^{44}$ may experience selection for poorly adapted codons ${ }^{45}$ or strong mRNA folding ${ }^{46}$. Codon choice that promotes proper nucleosome positioning is selectively advantageous in eukaryotes, especially in 5' regions ${ }^{47}$. And, finally, in mammals, synonymous mutations near an intron-exon boundary can create spurious splice sites or disrupt splicing control elements ${ }^{4,48}$, causing disease $\mathrm{e}^{4}$. This phenomenon helps to explain the reduced rate of synonymous substitutions and SNP density near splicing control elements ${ }^{49,50}$. Selection for proper splicing also extends to D. melanogaster, and sequence variation suggests that it is probably an even stronger force than translational selection in shaping codon usage near intron-exon boundaries ${ }^{51}$.

Although important, the mechanisms of intragenic codon-usage variation described above are typically restricted to specific taxa or special classes of sites. Recent studies have argued for three mechanisms that produce systematic variation in codon usage across the sites in a gene in a diverse range of species. 
Ribosomal pausing

A temporary arrest of the ribosome during

translation elongation.

Effective population size

The number of individuals in a population that produce viable offspring

Biased gene conversion A recombination event in which one variant of genomic sequence is preferentially 'copied and pasted' onto another one.

Fourfold degenerate sites Positions within the coding sequence of a gene at which all four nucleotides encode the same amino acid.

Shotgun proteomics Methods of quantifying protein levels in a complex sample, typically using mass spectrometry.
One of these mechanisms is selection against strong $5^{\prime}$ mRNA structure to facilitate translation initiation. mRNA structure near the $5^{\prime}$ end of a coding region is generally disadvantageous ${ }^{9}$ as it can inhibit ribosomal initiation $^{52,53}$ (FIG. 3a). Eyre-Walker and Bulmer proposed selection against mRNA structure to explain a trend towards reduced codon adaptation in the $5^{\prime}$ region of E. coli genes and a corresponding reduced rate of synonymous substitutions across divergent species $^{54}$. More recently, following similar observations in E. coli ${ }^{55}$, $\mathrm{Gu}$ et al. demonstrated a broad trend in all sequenced prokaryotes and eukaryotes towards reduced mRNA stability near the translation initiation sites of genes, especially for GC-rich genes ${ }^{56}$. This study relied on computational predictions of mRNA structure in short windows; combined with large-scale experimental studies (see below), this work suggests a systematic role for selection on mRNA structure in shaping codon usage in the first 30-60 nucleotides of genes.

Tuller et al. ${ }^{57}$ recently described a second, systematic trend in the pattern of intragenic codon usage: a 'ramp' of poorly adapted codons in the first 90-150 nucleotides of genes, which had earlier been observed in bacteria, yeast and fruitflies ${ }^{58,59}$. This pattern has been preserved across divergent species even when tRNA pools (estimated from gene copy numbers) have changed ${ }^{57}$. A ramp of poorly adapted codons presumably slows elongation at the start of a gene, which may provide several physiological benefits. Slow $5^{\prime}$ elongation is predicted to reduce the frequency of ribosomal traffic jams towards the $3^{\prime}$ end ${ }^{57,60}$, thus reducing the cost of wasted ribosomes and of spontaneous or collision-induced abortions. Alternatively, a ramp of slow elongation may facilitate recruitment of

\section{Box 2 | Mammals are different}

Intragenomic patterns of codon usage in mammals are markedly different from those in other taxa. Selective mechanisms were initially ruled out for humans on the basis of their small effective population size, which limits the efficacy of selection ${ }^{4}$. Moreover, the most obvious pattern of gene-to-gene codon-usage variation in mammals arises not from selection but from large-scale variation in the GC content - that is, the isochores ${ }^{116}$. Isochores themselves are probably caused by processes that are primarily related to recombination and repair such as biased gene conversion ${ }^{117}$.

Over the past decade, however, researchers have identified several sources of potentially strong selection on synonymous mutations in mammals, a trend that was highlighted by Hurst and others ${ }^{4}$. Some of these observations fit within the classical model of translational selection, for example, the presence of a weak but positive relationship between gene expression and codon bias ${ }^{118,119}$, especially after accounting for the local GC content ${ }^{120}$. But studies comparing expression levels to codon adaptation (that is, to tRNA abundances) have been contradictory ${ }^{33,36,119}$. Researchers have also observed significant differences in codon usage between genes specifically expressed in several different tissues ${ }^{121}$, as well as variation in relative tRNA abundances by tissue type ${ }^{122}$. However, there is little evidence for systematic variation associated with tissue type ${ }^{123}$, and the quantification of mammalian tRNAs, which contain numerous nucleotide modifications, is still relatively noisy ${ }^{122}$.

Instead, researchers have identified other mechanistic explanations for codon-usage variation in mammals aside from translational selection. One possibility is selection for the overall stability of mRNA transcripts ${ }^{124,125}$ through a skew towards $C$ at fourfold degenerate sites. In mice, computational analyses suggest that such skews have been selected to promote mRNA stability ${ }^{124}$. Moreover, several diseases arise from mutations that disrupt mRNA structure ${ }^{4,126}$, providing a clear target of selection. Another possibility related to splicing control is described in the main text. chaperone proteins to the emergent peptide ${ }^{61}$. Other explanations, unrelated to elongation rate, are also plausible such as weaker selection for accurate translation near the start of a gene, where missense and nonsense errors would be less costly ${ }^{24,59}$. The earliest interpretation of unusual $5^{\prime}$ codon usage posited selection to increase the initiation rate ${ }^{9}$; interestingly, the $5^{\prime}$ region of poorly adapted codons identified by Tuller et al. overlaps significantly with the region in which synonymous codon choice systematically reduces mRNA stability ${ }^{54-56,58}$. It remains unclear which selective mechanisms are primarily responsible for the unusual and nearly universal pattern of 5' codon usage. Multiple mechanisms may certainly operate in different genes; however, it is unclear why a single gene should experience selection both to increase its rate of ribosomal initiation ${ }^{9}$ and to reduce the subsequent rate of its early elongation ${ }^{57}$.

Cannarozzi et al. ${ }^{62}$ recently exposed a third, novel pattern of intragenic codon usage in eukaryotes: the reuse or autocorrelation of codons across a gene sequence, driven, they argue, to improve elongation efficiency through tRNA recycling. If a recently used tRNA molecule is bound to the ribosome, or if it diffuses slowly compared to ribosomal progression and re-acylation ${ }^{63}$, then it would be efficient to re-use the same tRNA molecule for subsequent incorporations of the same amino acid. This physical model predicts selection for using the same codon or, more generally, a codon that is read by the same tRNA species, at nearby sites in a gene that encode the same amino acid. Indeed, Cannarozzi et al. observed significant autocorrelation of codons across gene sequences in most eukaryotes, especially in genes that are rapidly upregulated in response to stress. Of course, autocorrelation would also be predicted if all sites in a gene independently experience pressure for biased codon usage, for example, to match the global pool of tRNAs. To control for overall codon usage, Cannarozzi et al. compared the degree of autocorrelation in actual gene sequences to gene sequences that had been reshuffled at random, finding more autocorrelation on average in the unshuffled genes, although only marginally so. More convincingly, they observed that autocorrelation is strongest for iso-accepting codons of rare tRNAs in highly expressed genes, which is predicted by the tRNArecycling hypothesis but not by a selective pressure that applies at all sites independently.

\section{Measurements of endogenous expression}

Recent developments in mass spectrometry and fluorescence microscopy allow large-scale measurements of endogenous protein levels ${ }^{64-66}$. Together with techniques for quantifying ribosomal occupancy ${ }^{67}$ and measuring elongation dynamics ${ }^{68}$, these advances provide a spectacularly detailed account of basic cellular processes, with implications for our understanding of codon biases.

Variation in protein/mRNA ratios. Shotgun proteomics have revealed an extensive role for post-transcriptional processes in determining eventual protein levels in bacteria, yeast ${ }^{69}$, worms ${ }^{70}$, fruitflies ${ }^{70}$ and especially mammals ${ }^{65,66}$. Whereas the imperfect correlations between protein and 
a
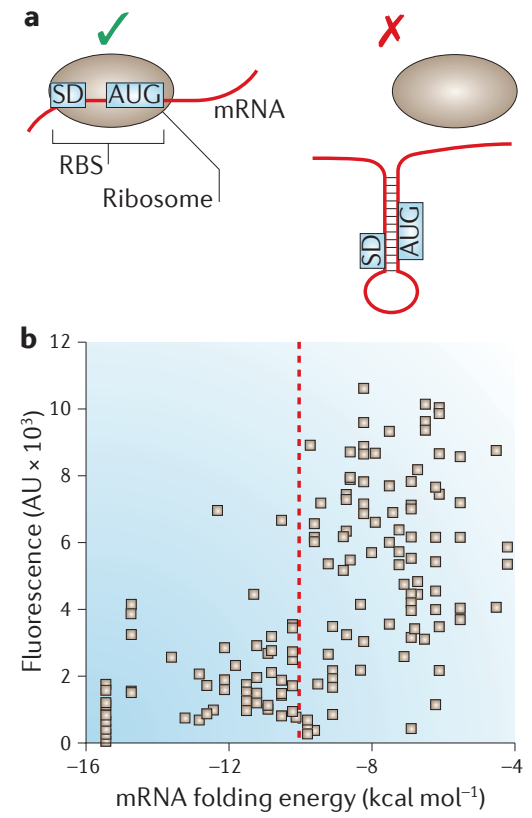
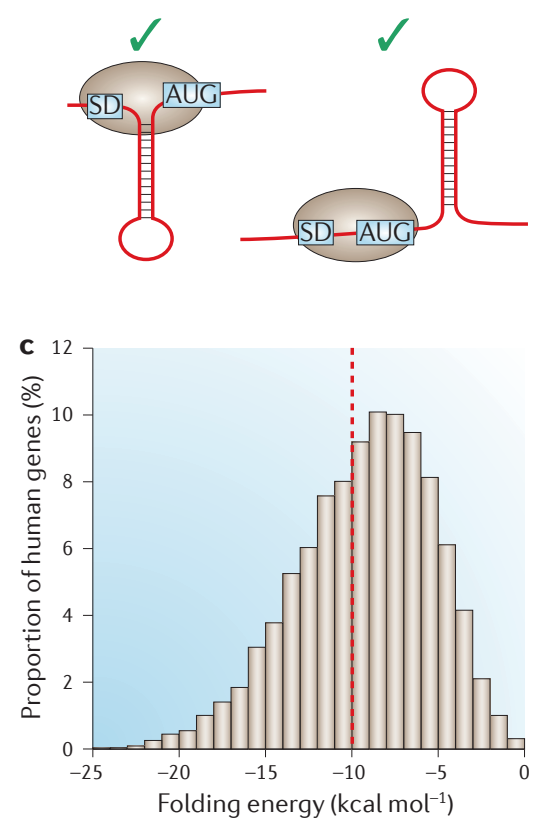

Figure 3 | Effects of mRNA secondary structure on translation initiation in bacteria. a | Structure in the ribosome binding site (RBS) usually inhibits initiation. However, initiation can occur when the structured element is positioned between the Shine-Dalgarno sequence (SD) and the start codon (AUG) ${ }^{129}$, or 15 nucleotides downstream of the start codon ${ }^{130,131} \cdot \mathbf{b}$ | Synonymous mutations in the region from nucleotide -4 to +37 of a GFP gene alter the predicted folding energies by up to $12 \mathrm{kcal} \mathrm{mol}^{-1}$. A $5^{\prime} \mathrm{mRNA}$ folding energy of below $-10 \mathrm{kcal} \mathrm{mol}^{-1}$ strongly inhibits GFP expression in Escherichia coli ${ }^{55}$. c | More than $40 \%$ of human genes have predicted 5' folding energies below the $-10 \mathrm{kcal} \mathrm{mol}^{-1}$ threshold and are therefore expected to express poorly in E. coli without modification. AU, arbitrary units. Part $\mathbf{b}$ is modified, with permission, from REF. 55 @ (2009) American Association for the Advancement of Science.

Codon adaptation index A measure of similarity between the codon usage of a gene and the average codon usage of highly expressed genes in a species.

\section{RNA-seq}

Quantitative analysis of RNA in a complex sample by high-throughput sequencing.

Ribosomal footprint A fragment of mRNA that is protected by ribosomes from nuclease digestion in a ribosomal-profiling experiment.

Upstream ORFs ORFs that are located $5^{\prime}$ from the primary ORF. They are thought to inhibit translation of the primary ORF.
mRNA levels $\left(\mathrm{R}^{2} \approx 47-77 \%\right.$ in E. coli ${ }^{65,71}, 73 \%$ in yeast ${ }^{65}$ and $29 \%$ in humans ${ }^{66}$ ) may previously have been seen as measurement noise, researchers have since attributed much of the variation in protein/mRNA ratios to sequence-derived characteristics of genes. In a recent study in human cells ${ }^{66}$, the strongest correlates of steady-state protein levels, controlling for mRNA levels, were coding-sequence length (reflecting the fact that longer transcripts are less stable $e^{72}$ or slower to initiate ${ }^{73}$ ), amino acid content (reflecting the variable costs associated with synthesizing different amino acids, or variable rates of protein degradation) and predicted $5^{\prime} \mathrm{mRNA}$ structure (reflecting lower initiation rates when the $5^{\prime}$ structure is strong). Importantly, the codon adaptation index ${ }^{35}$, which correlates strongly with mRNA levels in yeast ${ }^{65}$ and weakly in humans ${ }^{66}$, shows little or no significant correlation with the amount of protein per mRNA molecule in either organism ${ }^{65,66}$; this suggests that codon adaptation does not significantly increase the protein yield from a given message, at least among endogenous genes ${ }^{74,75}$. It is important to note that steady-state protein levels are influenced by both protein production and protein degradation, so any variation in degradation rates unrelated to codon usage will further reduce the correlation between codon usage and protein/mRNA ratios.
Ribosomal footprints. Ingolia et al. recently devised a clever application of RNA-seq to quantify ribosomeprotected RNA fragments in a cell, thereby estimating 'ribosomal footprints' across the transcriptome ${ }^{67}$. This method has provided rich information about translational regulation and it has uncovered some startling phenomena, such as an abundance of upstream ORFs with non-AUG start codons. The footprint data in yeast show a greater mean density of ribosomes in the first 100-150 codons of genes, suggesting locally slow elongation; this is consistent with the observed presence of poorly adapted codons in the $5^{\prime}$ region ${ }^{58,59}$. There is also a significant negative correlation, genome-wide, between a transcript's ribosome density and the experimentally measured strength of mRNA structure near its start site ${ }^{76}$, suggesting that strong $5^{\prime}$ mRNA structure retards translational initiation and reduces the density of translating ribosomes.

Remarkably, on averaging data from all yeast genes, Tuller et al. ${ }^{37}$ also observed a negative correlation between predicted mRNA folding energy and ribosome density among the first 65 codons, suggesting that strong mRNA structure downstream of the start site retards translational elongation. This observation is surprising, given the helicase activity of translating ribosomes ${ }^{77}$; however, the correlation between the genome-wide average profiles of mRNA folding and ribosome density does not imply a correlation at the level of individual sites. Ingolia et al. also measured ribosomal footprints under amino acid starvation and found that one-third of yeast genes showed substantially increased or decreased translational efficiency in these conditions compared with controls ${ }^{67}$. A detailed parsing of the relationship between a gene's amino acid content and translational response to starvation may improve design principles for overexpressed heterologous genes, which often induce starvation ${ }^{78,79}$ (see below).

Translational efficiency. Notions of translational efficiency differ in the literature on gene expression. Ingolia et al. ${ }^{67}$ defined the translational efficiency of a gene as the number of bound ribosomes per mRNA molecule; by contrast, Tuller et al. ${ }^{37,57}$ and others defined efficiency as protein yield per mRNA molecule (that is, the ratio of protein abundance to mRNA abundance). The second definition is more relevant to issues of total protein synthesis, whereas the former definition may be more relevant to ribosomal availability and overall cellular fitness. These two notions of translational efficiency are only weakly correlated for endogenous genes $\left(\mathrm{R}^{2}<2.5 \%\right.$ comparing the data by Ingolia et al. ${ }^{67}$ to REF. 80 ), indicating that the density of ribosomes on a given mRNA molecule does not determine the amount of protein that is produced from it. Similarly, in yeast, a gene's codon adaptation index ${ }^{35}$ explains less than $3 \%$ of the variance in protein abundance per mRNA ${ }^{67}$. Both of these observations are consistent with the idea that, for most endogenous genes, the initiation is rate limiting for protein production ${ }^{38,39}$ and therefore determines the amount of protein produced from each message, regardless of ribosome density or codon adaptation ${ }^{7,9}$ (FIG. 2); 
however, this logic may not apply to overexpressed heterologous genes, which are described in the following section (FIG. 4).

\section{Measurements of heterologous expression}

Codon bias has a crucial role in heterologous gene expression. However, there is often a disconnection between technological and evolutionary studies of codon bias - a gap that partly reflects genuine differences between endogenous and heterologous situations. In many biotechnological applications, a transgene is massively overexpressed, accounting for up to $30 \%$ of the protein mass in cell. As a result, the principles that relate heterologous codon usage to protein levels may differ substantially from the endogenous case.

The idea that initiation generally limits translation may not apply to an overexpressed transgene whose mRNA accounts for a large proportion of total cellular mRNA. In such a case, inefficient use of ribosomes along the overexpressed mRNA may be sufficient to feed back and significantly deplete available ribosomes, thereby reducing initiation rates and retarding further heterologous protein production ${ }^{9}$ (FIG. 4). Thus, we might expect that the elongation effects of codon usage will influence protein yields per mRNA molecule for overexpressed genes. Nonetheless, we should not necessarily expect that the codons that are adapted to efficient elongation for endogenous genes will correspond to the efficient codons for heterologous genes, because overexpression causes amino acid starvation and concomitant alternations in the abundances of charged tRNAs ${ }^{78,79,81}$. Indeed, there was no significant correlation between codon adaptation ${ }^{35}$ and expression levels in two large-scale systematic experiments ${ }^{55,79}$. In fact, even endogenous genes that are essential during amino acid starvation such as amino acid biosynthetic enzymes preferentially use codons that are poorly

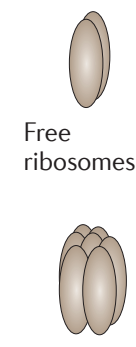

a Initiation limited, slow elongation

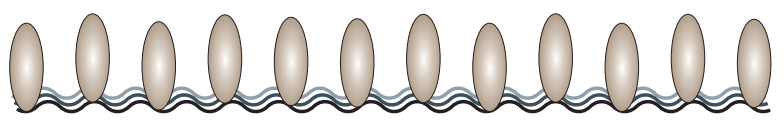

b Initiation limited, rapid elongation
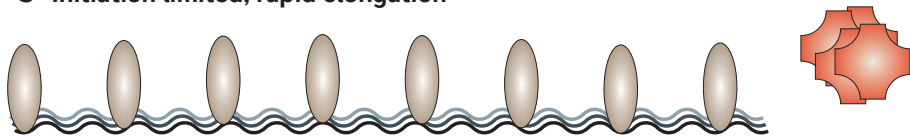

Figure 4 | The elongation rate may influence the rate of protein synthesis for an overexpressed gene. Unlike most endogenous genes, mRNA from an overexpressed transgene may account for a substantial proportion of total cellular mRNA. In this case, slow elongation (caused by poor codon adaptation to charged tRNA pools, say) can increase the density of bound ribosomes and thereby reduce the pool of available ribosomes in the cell. Such a depletion of available ribosomes will feed back to reduce the initiation rate of subsequent translating ribosomes on the message, thereby reducing the rate of protein synthesis. This is illustrated schematically by comparing overexpressed mRNAs with slow elongation (above) and rapid elongation (below), but identical initiation sequences. Thus, the relationship between codon adaptation and the rate of protein synthesis per mRNA molecule may differ for an overexpressed transgene compared to an endogenous gene (FIG. 2). adapted to the typical pool of charged tRNAs, but are well adapted to starvation-induced tRNA pools ${ }^{78,79}$.

Despite the complications described above, the field of codon optimization has traditionally focused on adjusting codon usage to match cellular tRNA abundances in standard conditions, disregarding other dimensions of bias. However, strategies are now changing. Several recent studies advocate for the role of global nucleotide content ${ }^{82,83}$, local mRNA folding ${ }^{55,84}$, codon pair bias ${ }^{85}$, a codon ramp ${ }^{57}$ or codon correlations ${ }^{62}$ in optimizing heterologous expression (TABLE 1).

Effects of codon adaptation on expression levels. Many studies show strong effects of rare codons on heterologous expression. In E. coli, stretches of rare AGA or AGG codons cause ribosome pausing and co-translational cleavage of mRNA ${ }^{86}$, ribosomal frameshifting ${ }^{87}$ or amino acid misincorporation ${ }^{88}$. Consistent with theoretical expectations, codons that are read by rare tRNAs can slow elongation by several fold ${ }^{89}$. And stretches of AGG codons near the ribosome-binding site (RBS) can reduce protein yields by obstructing translation initiation ${ }^{90}$. Although such studies are convincing, they usually address the effect of a subset of rare codons, often in long stretches, in E. coli cells; it is not known whether these principles can be applied in general.

Observations such as those above were quickly followed by efforts to adjust the global codon adaptation of transgenes to cellular tRNA abundances. Several approaches have been proposed: 'CAI maximization' replaces all codons by the most preferred codons in the target genome, but this could result in unbalanced charged tRNA pools'; 'codon harmonization' ${ }^{\text {'11 }}$ puts some non-preferred codons in positions that correspond to predicted protein domain boundaries; and 'codon sampling' adjusts the codon usage to reflect the overall usage in the target genome. In the absence of tRNA abundance estimates, codon frequencies in the target genome are sometimes used. It has also been suggested that codon usage should match the profile of charged tRNAs rather than total tRNAs ${ }^{79,81}$. The utility of codon adaptation approaches is still unclear, as they have not been systematically compared against each other, and several anecdotal studies argue both for (for example, REF. 92) and against (for example, REF. 93) their efficiency.

Codon adaptation algorithms typically optimize many sequence properties at once. This makes it difficult to determine which parameter causes observed differences in expression. In two recent multi-gene studies, between $60 \%$ and $70 \%$ of genes experienced increased expression upon codon optimization ${ }^{94,95}$, but whether this was a direct consequence of increased codon adaptation or other sequence properties is unclear. In our study of 154 synonymous variants of GFP, we observed no significant correlation between the codon adaption index ${ }^{35}$ and expression levels in E. coli $i^{55}$, but a weak positive correlation was later found using nonlinear regressions $s^{37,96}$. In any case, adaptation of codon usage is limited to species with pronounced and wellunderstood variation in tRNA concentrations, such as bacteria and yeast. 
Effects of nucleotide bias on expression levels. Nucleotide biases are pervasive in natural genes and have the potential to alter the interactions of mRNA with DNA, with proteins and with itself, thereby influencing RNA production, degradation and translation rates. Many of these effects are characterized, but this knowledge has yet to find its way into standard codon optimization procedures.
GC-rich mRNAs can form strong secondary structures, and, in bacteria, strong structure near the RBS prohibits initiation ${ }^{53,55,97}$ (FIC. 3b). As a result, more than $40 \%$ of human genes would be expected to express poorly when placed in E. coli without modification (Fig. 3c). Strong structure near the start codon reduces heterologous expression in yeast as well (G.K., unpublished observations), consistent with evolutionary analyses ${ }^{56}$.

\begin{tabular}{|c|c|c|c|c|c|c|}
\hline Parameter & Species & $\begin{array}{l}\text { Relationship } \\
\text { with expression }\end{array}$ & $\begin{array}{l}\text { Type of } \\
\text { evidence }\end{array}$ & Proposed mechanism & $\begin{array}{l}\text { Refs } \\
\text { (computation)* }\end{array}$ & $\begin{array}{l}\text { Refs } \\
\text { (function) }\end{array}$ \\
\hline \multicolumn{7}{|l|}{ Codon adaptation } \\
\hline CAI, FOP or tAl & All & $\begin{array}{l}\text { Complex } \\
\text { (see text) }\end{array}$ & Experimental & $\begin{array}{l}\text { Translation elongation rate } \\
\text { and accuracy }\end{array}$ & $\begin{array}{l}\mathrm{CAl}^{35}, \mathrm{FOP}^{28}, \\
\mathrm{tAl} l^{128}\end{array}$ & $\begin{array}{l}2,37,55 \\
81,94,96\end{array}$ \\
\hline Rare codon stretches & Bacteria & Negative & Experimental & $\begin{array}{l}\text { Translation elongation rate } \\
\text { and accuracy }\end{array}$ & & 90 \\
\hline $\begin{array}{l}\text { Rare codons between protein } \\
\text { domains }\end{array}$ & Bacteria & Complex & Experimental & $\begin{array}{l}\text { Translation elongation and } \\
\text { protein folding }\end{array}$ & & 91,132 \\
\hline $\begin{array}{l}\text { Frequency of starvation- } \\
\text { resistant codons }\end{array}$ & Bacteria & Positive & Experimental & $\begin{array}{l}\text { Translation elongation rate } \\
\text { and accuracy }\end{array}$ & 79,81 & 81 \\
\hline Frequency of abundant codons & All & Complex & Experimental & Unclear ${ }^{\ddagger}$ & 133 & 134 \\
\hline $\begin{array}{l}\text { mRNA stem-loops further } \\
\text { downstream }\end{array}$ & All & Complex & Experimental & Translation elongation & 135 & 46,84 \\
\hline \multicolumn{7}{|l|}{ Regulatory motifs } \\
\hline Transcription terminators & Bacteria & Negative & Experimental & Transcription & RNAMotif $^{136}$ & \\
\hline RNase E sites & Bacteria & Negative & Experimental & mRNA stability & 137 & \\
\hline miRNA target sites & Eukaryotes & Negative & Experimental & Translation, mRNA stability & TargetScan ${ }^{138}$ & \\
\hline Various mRNA regulatory elements & All & Complex & Theoretical & Translation, mRNA stability & TransTerm $^{139}$ & \\
\hline \multicolumn{7}{|l|}{ Nucleotide bias } \\
\hline High GC content, low A content & Mammals & Positive & Experimental & $\begin{array}{l}\text { Transcription, mRNA } \\
\text { processing, mRNA export }\end{array}$ & 140,141 & $82,83,101$ \\
\hline Codon correlation & Eukaryotes & Positive & Experimental & Translation elongation rate & 62 & 62 \\
\hline Unknown & All & Complex & Experimental & Protein-folding efficiency & & 107 \\
\hline Unknown & Eukaryotes & Complex & Theoretical & Splicing regulation & & 4,142 \\
\hline Unknown & All & Complex & Experimental & $\begin{array}{l}\text { Protein post-translational } \\
\text { modification }\end{array}$ & 44 & 44 \\
\hline Replication strand nucleotide bias & $\begin{array}{l}\text { Bacteria, } \\
\text { mitochondria }\end{array}$ & Unknown & None & Unknown & & \\
\hline CTAG avoidance & Bacteria & Unknown & None & Restriction avoidance & 143 & 143 \\
\hline
\end{tabular}

Note, this table lists coding-sequence-derived parameters that can be changed by synonymous mutations. Other parameters may be important for expression (for example, the identity of the amino-terminal amino acid or the length of the sequence) but they require non-synonymous changes. ${ }^{\star} \mathrm{Generic}$ tools to calculate some of these parameters: codonW ${ }^{140}$ (http://codonw.sourceforge.net/), INCA ${ }^{141}$ and GeneDesigner ${ }^{144}$. 垚e frequency of abundant codons is highly correlated with GC content in mammals. ${ }^{\S}$ The codon ramp is predicted to decrease the cost of translation, which may indirectly influence expression levels. CAI, codon adaptation index; FOP, frequency of 'optimal' codons; miRNA, microRNA; tAI, tRNA adaptation index. 
No such effect has been described in mammals; on the contrary, high GC content generally increases expression levels in mammalian cells (see below). However, a strong mRNA hairpin in the coding sequence has been reported to interfere with translation in mammalian cells ${ }^{84}$, and strong hybrids between RNA and DNA (the R-loops) may interfere with transcription ${ }^{98}$.

GC-poor mRNAs are unlikely to fold strongly, but they often carry other sequence elements that limit expression. For example, low GC content is commonly believed to limit the expression of Plasmodium falciparum genes in E. coli, although the mechanisms are unknown. Such mRNAs may be targets for RNase E, which cleaves AU-rich sequences with low sequence specificity ${ }^{99}$. The situation is slightly clearer in mammals, in which low GC content (or high A content) has been shown to reduce expression ${ }^{82,83}$. This effect is common knowledge in virology, as HIV and human papilloma virus (HPV) genes are poorly expressed in human cells unless the gene sequences are optimized ${ }^{100,101}$. The rate-limiting step in these cases may be transcription or nuclear RNA export $^{82,83}$, which is consistent with the efficient expression of GC-poor genes in cytoplasmic transcription systems based on the vaccinia virus ${ }^{101}$.

Little is known about the functional consequences of replication strand-related bias or CTAG avoidance, which are common in prokaryotes. High $\mathrm{CpG}$ content was reported to correlate with high expression in mammalian cells ${ }^{102}$, possibly by altering the distribution of nucleosomes on DNA.

Other effects of synonymous mutations on expression levels. Other examples of synonymous mutations influencing expression have been described as primarily anecdotal observations. In $E$. coli, overrepresented codon pairs ${ }^{103}$ were proposed to decrease translation elongation rates ${ }^{104}$, although this conclusion was later disputed ${ }^{105}$. In an attempt to produce attenuated strains, Coleman et al. ${ }^{85}$ partially de-optimized codon pairs in the poliovirus genome and observed a reduction in protein yield of several fold and a reduction in viral infectivity of 1,000 -fold in mammalian cells. A version of GFP with autocorrelated codon usage showed 30\% lower ribosome density in yeast, suggesting faster elongation, than a version with anticorrelated codon usage ${ }^{62}$. And a synonymous mutation in the human multidrug-resistance protein 1 (MDR1) gene was proposed to influence mRNA stability ${ }^{106}$ or protein folding and substrate specificity ${ }^{107}$. These observations are all intriguing and form important avenues for future systematic studies to determine their molecular bases.

\section{Conclusions}

Recent years have begun to see a convergence of experimental work on endogenous and heterologous gene expression, as both types of studies take advantage of high-throughput, quantitative techniques. Heterologous studies using large libraries of random or unbiased synonymous sequence variation ${ }^{55,81,97}$ are especially important for uncovering and comparing general rules to optimize expression. By contrast, relatively small-scale studies based on preconceived notions of 'optimized' codon usage do not provide sufficient power to distinguish among alternative mechanisms, nor do they allow us to discover any new mechanisms that increase expression. Heterologous studies will be complemented by endogenous measurements of initiation and elongation dynamics, and their effects on protein synthesis as a function of a gene's amino acid content and transcript level.

In the short term, there will be a trade-off between gaining predictive power for transgene optimization and deducing the underlying mechanisms that link codon usage and gene expression. High-dimensional, statistical regressions applied to large libraries of synonymous genes ${ }^{81,96}$ provide a principled, effective means of increasing heterologous expression. Such techniques are increasingly valuable in applied contexts in which high expression is required - such as viral-delivered gene therapies ${ }^{108,109}$ — but they do not generally identify molecular mechanisms. Our hope, over the long term, is that cross-fertilization between biotechnological and molecular biological studies will elucidate effective strategies for designing transgenes, as well as the mechanistic principles that underlie their expression.
1. Zuckerkandl, E. \& Pauling, L. Molecules as documents of evolutionary history. J. Theor. Biol. 8, 357-366 (1965).

2. Gustafsson, C., Govindarajan, S. \& Minshull, J. Codon bias and heterologous protein expression. Trends Biotechnol. 22, 346-353 (2004).

3. Hershberg, R. \& Petrov, D. A. Selection on codon bias Annu. Rev. Genet. 42, 287-299 (2008)

4. Chamary, J. V., Parmley, J. L. \& Hurst, L. D. Hearing silence: non-neutral evolution at synonymous sites in mammals. Nature Rev. Genet 7, 98-108 (2006).

An excellent Review of the many surprisingly strong effects of synonymous mutations on splicing.

5. Duret, L. Evolution of synonymous codon usage in metazoans. Curr. Opin. Genet. Dev. 12, 640-649 (2002).

6. Sharp, P. M., Averof, M., Lloyd, A. T., Matassi, G. \& Peden, J. F. DNA sequence evolution: the sounds of silence. Philos. Trans. R. Soc. Lond. B 349 , 241-247 (1995)

7. Andersson, S. G. \& Kurland, C. G. Codon preferences in free-living microorganisms. Microbiol. Rev. $\mathbf{5 4}$ 198-210 (1990).

A classic, must-read paper that has framed the field of codon-usage adaptation.
8. Kimura, M. A simple method for estimating evolutionary rates of base substitutions through comparative studies of nucleotide sequences. J. Mol Evol 16, 111-120 (1980)

9. Bulmer, M. The selection-mutation-drift theory of synonymous codon usage. Genetics 129, 897-907 (1991).

A foundational study of both the population genetics that underlie codon bias and the biophysics of translation. This paper emphasizes that, for endogenous genes, elongation speed wil not generally influence protein yield per mRNA.

10. Chen, S. L., Lee, W., Hottes, A. K., Shapiro, L. \& McAdams, H. H. Codon usage between genomes is constrained by genome-wide mutational processes. Proc. Natl Acad. Sci. USA 101, 3480-3485 (2004).

11. Hurst, L. D. \& Merchant, A. R. High guanine-cytosine content is not an adaptation to high temperature: a comparative analysis amongst prokaryotes. Proc. R. Soc. Lond. B 268, 493-497 (2001).

12. Fedorov, A., Saxonov, S. \& Gilbert, W. Regularities of context-dependent codon bias in eukaryotic genes. Nucleic Acids Res. 30, 1192-1197 (2002).
13. Hildebrand, F., Meyer, A. \& Eyre-Walker, A. Evidence of selection upon genomic GC-content in bacteria. PLoS Genet. 6, e1001107 (2010).

14. Morton, B. R. Selection at the amino acid level can influence synonymous codon usage: implications for the study of codon adaptation in plastid genes. Genetics 159, 347-358 (2001).

15. Cambray, G. \& Mazel, D. Synonymous genes explore different evolutionary landscapes. PLOS Genet. 4 e 1000256 (2008).

16. Plotkin, J. B. \& Dushoff, J. Codon bias and frequencydependent selection on the hemagglutinin epitopes of influenza A virus. Proc. Natl Acad. Sci. USA 100 7152-7157 (2003)

17. Sharp, P. M. $\& \mathrm{Li}, \mathrm{W}$. H. The rate of synonymous substitution in enterobacterial genes is inversely related to codon usage bias. Mol. Biol. Evol. 4 , 222-230 (1987).

18. Eyre-Walker, A. \& Bulmer, M. Synonymous substitution rates in enterobacteria. Genetics 140, 1407-1412 (1995).

19. Francino, M. P. \& Ochman, H. Deamination as the basis of strand-asymmetric evolution in transcribed Escherichia coli sequences. Mol. Biol. Evol. 18 1147-1150 (2001) 
20. Majewski, J. Dependence of mutational asymmetry on gene-expression levels in the human genome. Am. J. Hum. Genet. 73, 688-692 (2003).

21. Duret, L. \& Mouchiroud, D. Expression pattern and, surprisingly, gene length shape codon usage in Caenorhabditis, Drosophila, and Arabidopsis. Proc. Natl Acad. Sci. USA 96, 4482-4487 (1999).

22. Akashi, H. Synonymous codon usage in Drosophila melanogaster: natural selection and translational accuracy. Genetics 136, 927-935 (1994). By quantifying the rates of synonymous substitutions at conserved and non-conserved positions in proteins, Akashi shows that such mutations influence translational accuracy in D. melanogaster.

23. Akashi, H. \& Schaeffer, S. W. Natural selection and the frequency distributions of "silent" DNA polymorphism in Drosophila. Genetics 146, 295-307 (1997).

24. Eyre-Walker, A. Synonymous codon bias is related to gene length in Escherichia coli: selection for translational accuracy? Mol. Biol. Evol. 13, 864-872 (1996).

25. Stoletzki, N. \& Eyre-Walker, A. Synonymous codon usage in Escherichia coli: selection for translationa accuracy. Mol. Biol. Evol. 24, 374-381 (2007).

26 Drummond, D. A. \& Wilke, C. O. Mistranslationinduced protein misfolding as a dominant constraint on coding-sequence evolution. Cell 134, 341-352 (2008).

27. Zhou, T., Weems, M. \& Wilke, C. O. Translationally optimal codons associate with structurally sensitive sites in proteins. Mol. Biol. Evol. 26, 1571-1580 (2009).

28. Ikemura, T. Correlation between the abundance of Escherichia coli transfer RNAs and the occurrence of the respective codons in its protein genes: a proposal for a synonymous codon choice that is optimal for the $E$. coli translational system. J. Mol. Biol. 151, 389-409 (1981)

29. Ikemura, T. Correlation between the abundance of yeast transfer RNAs and the occurrence of the respective codons in protein genes. Differences in synonymous codon choice patterns of yeast and Escherichia coli with reference to the abundance of isoaccepting transfer RNAs. J. Mol. Biol. 158 573-597 (1982).

30. Post, L. E. \& Nomura, M. Nucleotide sequence of the intercistronic region preceding the gene for RNA polymerase subunit $\alpha$ in Escherichia coli. J. Biol. Chem. 254, 10604-10606 (1979).

31. Moriyama, E. N. \& Powell, J. R. Codon usage bias and tRNA abundance in Drosophila. J. Mol. Evol. 45, 514-523 (1997)

32. Duret, L. tRNA gene number and codon usage in the $C$. elegans genome are co-adapted for optimal translation of highly expressed genes. Trends Genet. 16, 287-289 (2000)

33. Kanaya, S., Yamada, Y., Kinouchi, M., Kudo, Y. \& Ikemura, T. Codon usage and tRNA genes in eukaryotes: correlation of codon usage diversity with translation efficiency and with CG-dinucleotide usage as assessed by multivariate analysis. J. Mol. Evol. 53, 290-298 (2001)

34. Sharp, P. M., Bailes, E., Grocock, R. J., Peden, J. F. \& Sockett, R. E. Variation in the strength of selected codon usage bias among bacteria. Nucleic Acids Res. 33, 1141-1153 (2005)

35. Sharp, P. M. \& Li, W. H. The codon Adaptation Index a measure of directional synonymous codon usage bias, and its potential applications. Nucleic Acids Res. 15, 1281-1295 (1987)

36. Lavner, Y. \& Kotlar, D. Codon bias as a factor in regulating expression via translation rate in the human genome. Gene 345, 127-138 (2005).

37. Tuller, T., Waldman, Y. Y., Kupiec, M. \& Ruppin, E. Translation efficiency is determined by both codon bias and folding energy. Proc. Natl Acad. Sci. USA 107, 3645-3650 (2010)

38. Bergmann, J. E. \& Lodish, H. F. A kinetic model of protein synthesis. Application to hemoglobin synthesis and translational control. J. Biol. Chem. 254, 11927-11937 (1979).

39. Mathews, M. B., Sonenberg, N. \& Hershey, J. W. B. (eds) Translational Control in Biology and Medicine (CHSL Press, New York, 2007).

40. Ozbudak, E. M., Thattai, M., Kurtser, I., Grossman, A. D. \& van Oudenaarden, A. Regulation of noise in the expression of a single gene. Nature Genet. 31, 69-73 (2002)

41 Fraser, H. B. Hirsh, A. E., Giaever, G., Kumm, J. \& Eisen, M. B. Noise minimization in eukaryotic gene expression. PLoS Biol. 2, e137 (2004).
42. Lawrence, J. G. \& Ochman, H. Molecular archaeology of the Escherichia coli genome. Proc. Natl Acad. Sci. USA 95, 9413-9417 (1998)

43. Karlin, S., Campbell, A. M. \& Mrazek, J. Comparative DNA analysis across diverse genomes. Annu. Rev. Genet. 32, 185-225 (1998).

44. Zhang, F., Saha, S., Shabalina, S. A. \& Kashina, A. Differential arginylation of actin isoforms is regulated by coding sequence-dependent degradation. Science 329, 1534-1537 (2010).

45. Thanaraj, T. A. \& Argos, P. Ribosome-mediated translational pause and protein domain organization Protein Sci. 5, 1594-1612 (1996)

46. Watts, J. M. et al. Architecture and secondary structure of an entire HIV-1 RNA genome. Nature 460, 711-716 (2009)

47. Warnecke, T., Batada, N. N. \& Hurst, L. D. The impact of the nucleosome code on proteincoding sequence evolution in yeast. PLoS Genet. 4 , e 1000250 (2008).

48. Eskesen, S. T., Eskesen, F. N. \& Ruvinsky, A. Natural selection affects frequencies of AG and GT dinucleotides at the 5' and 3' ends of exons. Genetics 167, 543-550 (2004).

49. Chamary J V. \& Hurst L. D. Biased codon usage near intron-exon junctions: selection on splicing enhancers, splice-site recognition or something else? Trends Genet. 21, 256-259 (2005)

50. Orban, T. I. \& Olah, E. Purifying selection on silent sites - a constraint from splicing regulation? Trends Genet. 17, 252-253 (2001).

51. Warnecke, T. \& Hurst, L. D. Evidence for a trade-off between translational efficiency and splicing regulation in determining synonymous codon usage in Drosophila melanogaster. Mol. Biol. Evol. 24 2755-2762 (2007)

52. Bettany, A. J. et al. 5'-secondary structure formation, in contrast to a short string of non-preferred codons, inhibits the translation of the pyruvate kinase mRNA in yeast. Yeast 5, 187-198 (1989).

53. de Smit, M. H. \& van Duin, J. Secondary structure of the ribosome binding site determines translational efficiency: a quantitative analysis. Proc. Natl Acad. Sci. USA 87, 7668-7672 (1990).

A thorough study, supported by a convincing theoretical model, of how mRNA folding affects translation initiation.

54. Eyre-Walker, A. \& Bulmer, M. Reduced synonymous substitution rate at the start of enterobacterial genes. Nucleic Acids Res. 21, 4599-4603 (1993).

55. Kudla, G., Murray, A. W., Tollervey, D. \& Plotkin, J. B. Coding-sequence determinants of gene expression in Escherichia coli. Science 324 255-258 (2009)

Uses libraries of synthetic genes to isolate the effects of synonymous mutations on expression. See also references 81 and 97 .

56. Gu, W., Zhou, T. \& Wilke, C. O. A universal trend of reduced mRNA stability near the translation-initiation site in prokaryotes and eukaryotes. PLoS Comput. Biol. 6, e1000664 (2010).

57. Tuller, T. et al. An evolutionarily conserved mechanism for controlling the efficiency of protein translation. Cell 141, 344-354 (2010).

Shows that rare codons at the beginning of genes could help prevent 'ribosomal traffic jams' See references 9,58 and 59 for an alternative interpretation.

58. Bulmer, M. Codon usage and intragenic position. J. Theor. Biol. 133, 67-71 (1988)

59. Oin, H., Wu, W. B., Comeron, J. M., Kreitman, M. \& Li, W. H. Intragenic spatial patterns of codon usage bias in prokaryotic and eukaryotic genomes. Genetics $168,2245-2260$ (2004).

60. Zhang, S., Goldman, E. \& Zubay, G. Clustering of low usage codons and ribosome movement. J. Theor. Biol. 170, 339-354 (1994)

61. Fredrick, K. \& Ibba, M. How the sequence of a gene can tune its translation. Cell 141, 227-229 (2010)

62. Cannarozzi, G. et al. A role for codon order in translation dynamics. Cell 141, 355-367 (2010).

63. Zouridis, H. \& Hatzimanikatis, V. Effects of codon distributions and tRNA competition on protein translation. Biophys. J. 95, 1018-1033 (2008)

64. Huh, W. K. et al. Global analysis of protein ocalization in budding yeast. Nature 425 686-691 (2003)
65. Lu, P. Vogel, C. Wang R Yao, X \& Marcotte, E. M. Absolute protein expression profiling estimates the relative contributions of transcriptional and translational regulation. Nature Biotech. 25 , 117-124 (2007)

66. Vogel, C. et al. Sequence signatures and mRNA concentration can explain two-thirds of protein abundance variation in a human cell line. Mol. Syst. Biol. 6, 400 (2010).

References 65 and 66 describe a quantitative proteomics approach that promises new insights into the coding-sequence determinants of protein levels.

67. Ingolia, N. T., Ghaemmaghami, S., Newman, J. R. \& Weissman, J. S. Genome-wide analysis in vivo of translation with nucleotide resolution using ribosome profiling. Science 324, 218-223 (2009). A clever method for mapping the positions of ribosomes on messages with unprecedented accuracy; an essential tool for the study of translation kinetics.

68. Uemura, S. et al. Real-time tRNA transit on single translating ribosomes at codon resolution. Nature 464, 1012-1017 (2010)

69. Futcher, B., Latter, G. I., Monardo, P., McLaughlin, C. S. \& Garrels, J. I. A sampling of the yeast proteome. Mol. Cell. Biol. 19, 7357-7368 (1999).

70. Schrimpf, S. P. et al. Comparative functional analysis of the Caenorhabditis elegans and Drosophila melanogaster proteomes. PLoS Biol. 7, e48 (2009).

71. Taniguchi, Y. et al. Quantifying E. coli proteome and transcriptome with single-molecule sensitivity in single cells. Science 329, 533-538.

72. Feng, L. \& Niu, D. K. Relationship between mRNA stability and length: an old question with a new twist. Biochem. Genet. 45, 131-137 (2007).

73. Arava, Y., Boas, F. E., Brown, P. O. \& Herschlag, D Dissecting eukaryotic translation and its control by ribosome density mapping. Nucleic Acids Res. 33 2421-2432 (2005).

74. Welch, M., Villalobos, A., Gustafsson, C. \& Minshull, J. You're one in a googol: optimizing genes for protein expression. J. R. Soc. Interface 6 (Suppl. 4), S467-S476 (2009).

75. Gouy, M. \& Gautier, C. Codon usage in bacteria correlation with gene expressivity. Nucleic Acids Res. 10, 7055-7074 (1982)

76. Kertesz, M. et al. Genome-wide measurement of RNA secondary structure in yeast. Nature 467, 103-107 (2010).

77. Takyar, S., Hickerson, R. P. \& Noller, H. F. mRNA helicase activity of the ribosome. Cell 120, 49-58 (2005).

78. Dittmar, K. A., Sorensen, M. A., Elf, J., Ehrenberg, M $\&$ Pan, T. Selective charging of tRNA isoacceptors induced by amino-acid starvation. EMBO Rep. 6 151-157 (2005)

79. Elf, J., Nilsson, D., Tenson, T. \& Ehrenberg, M. Selective charging of tRNA isoacceptors explains patterns of codon usage. Science 300, 1718-1722 (2003).

A detailed theoretical model of what happens to tRNA in cells under starvation conditions.

80. Ghaemmaghami, S. et al. Global analysis of protein expression in yeast. Nature 425, 737-741 (2003)

81. Welch, M. et al. Design parameters to control synthetic gene expression in Escherichia coli. PLOS ONE 4, e7002 (2009).

82. Kudla, G., Lipinski, L., Caffin, F., Helwak, A. \& Zylicz, M. High guanine and cytosine content increases mRNA levels in mammalian cells. PLoS Biol. 4, e 180 (2006).

83 Han, J. S., Szak, S. T. \& Boeke, J. D. Transcriptional disruption by the L1 retrotransposon and implications for mammalian transcriptomes. Nature 429, 268-274 (2004).

84. Nackley, A. G. et al. Human catechol-O-methyltransferase haplotypes modulate protein expression by altering mRNA secondary structure. Science 314, 1930-1933 (2006).

85. Coleman, J. R. et al. Virus attenuation by genomescale changes in codon pair bias. Science $\mathbf{3 2 0}$ 1784-1787 (2008).

86. Hayes, C. S., Bose, B. \& Sauer, R. T. Stop codons preceded by rare arginine codons are efficient determinants of SsrA tagging in Escherichia coli. Proc. Natl Acad. Sci. USA 99, 3440-3445 (2002).

87. Spanjaard, R. A. \& van Duin, J. Translation of the sequence AGG-AGG yields 50\% ribosomal frameshift. Proc. Natl Acad. Sci. USA 85, 7967-7971 (1988). 
88. Kramer, E. B. \& Farabaugh, P. J. The frequency of translational misreading errors in $E$. coli is largely determined by tRNA competition. RNA 13, 87-96 (2007).

89. Sorensen, M. A., Kurland, C. G. \& Pedersen, S. Codon usage determines translation rate in Escherichia coli. J. Mol. Biol. 207, 365-377 (1989).

90. Chen, G. F. \& Inouye, M. Suppression of the negative effect of minor arginine codons on gene expression; preferential usage of minor codons within the first 25 codons of the Escherichia coli genes. Nucleic Acids Res. 18, 1465-1473 (1990).

91. Angov, E., Hillier, C. J., Kincaid, R. L. \& Lyon, J. A Heterologous protein expression is enhanced by harmonizing the codon usage frequencies of the target gene with those of the expression host. PLOS ONE 3 , e2189 (2008)

92. Rosenberg, A. H., Goldman, E., Dunn, J. J., Studier, F. W. \& Zubay, G. Effects of consecutive AGG codons on translation in Escherichia coli, demonstrated with a versatile codon test system. J. Bacteriol. 175, 716-722 (1993).

93. Gursky, Y. G. \& Beabealashvilli, R. The increase in gene expression induced by introduction of rare codons into the $\mathrm{C}$ terminus of the template. Gene 148, 15-21 (1994).

94. Burgess-Brown, N. A. et al. Codon optimization can improve expression of human genes in Escherichia coli: a multi-gene study. Protein Expr. Purif. 59, 94-102 (2008).

95. Maertens, B. et al. Gene optimization mechanisms: a multi-gene study reveals a high success rate of full-length human proteins expressed in Escherichia coli. Protein Sci. 19, 1312-1326 (2010).

96. Supek, F. \& Smuc, T. On relevance of codon usage to expression of synthetic and natural genes in Escherichia coli. Genetics 185, 1129-1134 (2010).

97. Voges, D., Watzele, M., Nemetz, C., Wizemann, S. \& Buchberger, B. Analyzing and enhancing mRNA translational efficiency in an Escherichia coli in vitro expression system. Biochem. Biophys. Res. Commun. 318, 601-614 (2004).

98 El Hage, A., French, S. L., Beyer, A. L. \& Tollervey, D. Loss of topoisomerase I leads to R-loop-mediated transcriptional blocks during ribosomal RNA synthesis. Genes Dev. 24, 1546-1558 (2010)

99. McDowall, K. J., Lin-Chao, S. \& Cohen, S. N. A + U content rather than a particular nucleotide order determines the specificity of RNase E cleavage. J. Biol. Chem. 269, 10790-10796 (1994).

100. Nguyen, K. L. et al. Codon optimization of the HIV-1 vpu and vif genes stabilizes their mRNA and allows fo highly efficient Rev-independent expression. Virology 319, 163-175 (2004).

101. Sokolowski, M., Tan, W., Jellne, M. \& Schwartz, S mRNA instability elements in the human papillomavirus type $16 \mathrm{~L} 2$ coding region. J. Virol. 72, 1504-1515 (1998)

102. Bauer, A. P. et al. The impact of intragenic $\mathrm{CpC}$ content on gene expression. Nucleic Acids Res. 38, 3891-3908 (2010)

103. Gutman, G. A. \& Hatfield, G. W. Nonrandom utilization of codon pairs in Escherichia coli. Proc. Natl Acad. Sci. USA 86, 3699-3703 (1989).

104. Irwin, B., Heck, J. D. \& Hatfield, G. W. Codon pair utilization biases influence translational elongation step times. J. Biol. Chem. 270, 22801-22806 (1995).

105. Cheng, L. \& Goldman, E. Absence of effect of varying Thr-Leu codon pairs on protein synthesis in a T7 system. Biochemistry 40, 6102-6106 (2001).

106. Wang, D., Johnson, A. D., Papp, A. C., Kroetz, D. L. $\&$ Sadee, W. Multidrug resistance polypeptide 1 (MDR 1, ABCB 1) variant $3435 \mathrm{C}>\mathrm{T}$ affects mRNA stability. Pharmacogenet. Genomics $\mathbf{1 5}$, 3693-3704 (2005).

107. Kimchi-Sarfaty, C. et al. A "silent" polymorphism in the MDR 1 gene changes substrate specificity. Science $315,525-528$ (2007).
108. Foster, H. et al. Codon and mRNA sequence optimization of microdystrophin transgenes improves expression and physiological outcome in dystrophic $m d x$ mice following AAV2/8 gene transfer. Mol. Ther. 16, 1825-1832 (2008)

109. Arruda, V. R. et al. Peripheral transvenular delivery of adeno-associated viral vectors to skeletal muscle as a novel therapy for hemophilia B. Blood 115 4678-4688.

110. Fuglsang, A. The relationship between palindrome avoidance and intragenic codon usage variations: a Monte Carlo study. Biochem. Biophys. Res. Commun. 316, 755-762 (2004).

111. Drummond, D. A. \& Wilke, C. O. The evolutionary consequences of erroneous protein synthesis. Nature Rev. Genet. 10, 715-724 (2009). A readable Review that summarizes the various types of errors that occur in protein synthesis, many of which are directly related to codon usage.

112. Akashi, H., Kliman, R. M. ¿ Eyre-Walker, A. Mutation pressure, natural selection, and the evolution of base composition in Drosophila. Genetica 102-103. 49-60 (1998).

113. Marais, G. \& Duret, L. Synonymous codon usage, accuracy of translation, and gene length in Caenorhabditis elegans. J. Mol. Evol. 52 , 275-280 (2001)

114. Higgs, P. G. \& Ran, W. Coevolution of codon usage and tRNA genes leads to alternative stable states of biased codon usage. Mol. Biol. Evol. 25 2279-2291 (2008)

115. Shah, P. \& Gilchrist, M. Effect of correlated tRNA abundances on translation errors and evolution of codon usage bias. PLoS Genet. 6, e 1001128 (2010).

116. Bernardi, G. et al. The mosaic genome of warm-blooded vertebrates. Science 228, 953-958 (1985)

117. Galtier, N., Piganeau, G., Mouchiroud, D. \& Duret, L. GC-content evolution in mammalian genomes: the biased gene conversion hypothesis. Genetics 159 907-911 (2001).

118. Urrutia, A. O. \& Hurst, L. D. The signature of selection mediated by expression on human genes. Genome Res. 13, 2260-2264 (2003).

119. Comeron, J. M. Selective and mutational patterns associated with gene expression in humans: influences on synonymous composition and intron presence. Genetics 167, 1293-1304 (2004)

120. Karlin, S. \& Mrazek, J. What drives codon choices in human genes? J. Mol. Biol. 262, 459-472 (1996).

121. Plotkin, J. B., Robins, H. \& Levine, A. J. Tissue-specific codon usage and the expression of human genes. Proc. Natl Acad. Sci. USA 101, 12588-12591 (2004)

122. Dittmar, K. A., Goodenbour, J. M. \& Pan, T. Tissue-specific differences in human transfer RNA expression. PLoS Genet. 2, e221 (2006).

123. Semon, M., Lobry, J. R. \& Duret, L. No evidence for tissue-specific adaptation of synonymous codon usage in human. Mol. Biol. Evol. 23, 523-529 (2005)

124. Chamary, J. V. \& Hurst, L. D. Evidence for selection on synonymous mutations affecting stability of mRNA secondary structure in mammals. Genome Biol. 6 R75 (2005).

125. Seffens, W. $₫$ Digby, D. mRNAs have greater negative folding free energies than shuffled or codon choice randomized sequences. Nucleic Acids Res. 27, 1578-1584 (1999).

126. Duan, J. et al. Synonymous mutations in the human dopamine receptor D2 (DRD2) affect mRNA stability and synthesis of the receptor. Hum. Mol. Genet. 12, 205-216 (2003).

127. Sharp, P. M., Tuohy, T. M. \& Mosurski, K. R. Codon usage in yeast: cluster analysis clearly differentiates highly and lowly expressed genes. Nucleic Acids Res. 14, 5125-5143 (1986)

128. dos Reis, M., Savva, R. \& Wernisch, L. Solving the riddle of codon usage preferences: a test for translational selection. Nucleic Acids Res. 32 , 5036-5044 (2004).
129. Nivinskas, R., Malys, N Klausa V Vaiskunaite, R. \& Gineikiene, E. Post-transcriptional control of bacteriophage T4 gene 25 expression: mRNA secondary structure that enhances translational initiation. J. Mol. Biol. 288, 291-304 (1999).

130. Kozak, M. Downstream secondary structure facilitates recognition of initiator codons by eukaryotic ribosomes. Proc. NatI Acad. Sci. USA 87, 8301-8305 (1990)

131. Paulus, M., Haslbeck, M. \& Watzele, M. RNA stem-loop enhanced expression of previously non-expressible genes. Nucleic Acids Res. 32, e78 (2004).

132. Zhang, G., Hubalewska, M. \& Ignatova, Z. Transient ribosomal attenuation coordinates protein synthesis and co-translational folding. Nature Struct. Mol. Biol. 16, 274-280 (2009)

133. Nakamura, Y., Gojobori, T. \& Ikemura, T. Codon usage tabulated from international DNA sequence databases: status for the year 2000. Nucleic Acids Res. 28, 292 (2000).

134. Zolotukhin, S., Potter, M., Hauswirth, W. W., Guy, J. ¿ Muzyczka, N. A "humanized" green fluorescent protein cDNA adapted for high-level expression in mammalian cells. J. Virol. 70, 4646-4654 (1996)

135. Markham, N. R. \& Zuker, M. DINAMelt web server for nucleic acid melting prediction. Nucleic Acids Res. 33, W577-W581 (2005)

136. Lesnik, E. A. et al. Prediction of rho-independent transcriptional terminators in Escherichia coli. Nucleic Acids Res. 29, 3583-3594 (2001)

137. Bernstein, J. A., Khodursky, A. B., Lin, P. H., Lin-Chao, S. \& Cohen, S. N. Global analysis of mRNA decay and abundance in Escherichia coli at single-gene resolution using two-color fluorescent DNA microarrays. Proc. Natl Acad. Sci. USA 99, 9697-9702 (2002).

138. Lewis, B. P., Shih, I. H., Jones-Rhoades, M. W., Bartel, D. P. \& Burge, C. B. Prediction of mammalian microRNA targets. Cell 115, 787-798 (2003).

139. Jacobs, G. H. et al. Transterm: a database to aid the analysis of regulatory sequences in mRNAs. Nucleic Acids Res. 37, D72-D76 (2009)

140. Peden, J. F. Analysis of Codon Usage. Thesis, Dept of Genetics, Univ. Nottingham (1999).

141. Supek, F. \& Vlahovicek, K. INCA: synonymous codon usage analysis and clustering by means of selforganizing map. Bioinformatics 20, 2329-2330 (2004).

142. Pagani, F., Raponi, M. \& Baralle, F. E. Synonymous mutations in CFTR exon 12 affect splicing and are not neutral in evolution. Proc. Natl Acad. Sci. USA 102, 6368-6372 (2005)

143. Burge, C., Campbell, A. M. \& Karlin, S. Over- and under-representation of short oligonucleotides in DNA sequences. Proc. Natl Acad. Sci. USA 89, 1358-1362 (1992).

144. Villalobos, A., Ness, J. E., Gustafsson, C., Minshull, J. \& Govindarajan, S. Gene Designer: a synthetic biology tool for constructing artificial DNA segments. BMC Bioinformatics 7, 285 (2006).

\section{Acknowledgements}

We thank L. Hurst and C. Wilke for helpful discussions. We apologize to those whose work we were unable to cite because of space constraints. G.K. acknowledges funding from the Wellcome Trust. J.B.P. acknowledges support from the Burroughs Wellcome Fund the David and Lucile Packard Foundation, the James S. McDonnell Foundation, the Alfred P. Sloan Foundation, the Defense Advanced Research Projects Agency (HR0011-05-1-0057) and the US National Institute of Allergy and Infectious Diseases (2U54AI057168).

\section{Competing interests statement}

The authors declare no competing financial interests.

FURTHER INFORMATION

Joshua Plotkin's homepage: http://mathbio.sas.upenn.edu

ALL LINKS ARE ACTIVE IN THE ONLINE PDF 